\title{
The LAD Methods in WLAN Indoor Multipath Channels
}

\author{
Johanna Vartiainen, Janne Lehtomäki and Risto Vuohtoniemi \\ University of Oulu \\ Centre for Wireless Communications (CWC) \\ P.O. Box 4500, FI-90014 University of Oulu, FINLAND
}

\begin{abstract}
Spectrum sensing can be used to find free channels in cognitive radio systems. The localization algorithm based on double-thresholding (LAD) signal detection methods have been proposed to be used for spectrum sensing. Even though being widely studied, the performance of the LAD methods has not been studied in realistic channels. In this paper, ETSI BRAN/WLAN channel models $B$ and $C$ are used to study the performance of the LAD methods in the presence of realistic multipath, fading and Doppler effects in order to get statistical information about their performance. Measurements at the WLAN bands verify that the LAD methods have a good performance even in multipath situations.
\end{abstract}

Index Terms-narrowband signal, signal detection, energy measurement, multipath channels.

\section{INTRODUCTION}

Cognitive radio has been proposed to reorganize the whole old-fashioned spectrum utilization [1], [2], [3]. In Mitola's original idea [4], [5], cognitive radio system (CRS) allows secondary (unlicensed) users to use free frequency channels if they do not cause any interference to primary (licensed) users. The problem is how to find these free channels. As primary user signals can be assumed to be known or can be easily found using geolocation and/or databases, it is much more challenging to find signals from other secondary users because there is no a priori knowledge about their frequency location or even their signal types or other parameters. Spectrum sensing is one possibility to find out free space without any knowledge about other signals [6], [7], [8]. Notwithstanding, it is very important to the operation of the whole cognitive radio that secondary users have some knowledge about each other. Un-organized operation where secondary users cumulate to the same channel at the same time interfering or even preventing others transmission can lead to chaos which can jam the whole system. Thus, it can be said that spectrum sensing does have a future. Sensing can be performed using some signal detection method [9], [10], [9], [11]. It is advantageous if the used detection method is blind with relation to signal type and its parameters, so it is possible to detect several kind of signals with one method.

The research leading to these results was derived from the European Community's Seventh Framework Programme (FP7) under Grant Agreement number 248454 (QoSMOS).
The localization algorithm based on double-thresholding (LAD) signal detection methods that use the forward consecutive mean excision (FCME) algorithm have been widely studied and their performance has been analyzed, evaluated and compared in several papers, see e.g. [12], [13], [14] and references therein. The LAD methods include the original LAD method and its extensions called the LAD with adjacent clustering (ACC) and two-dimensional (2-D) LAD methods. The LAD methods are able to detect all kind of signals that cover at most $90 \%$ of the studied band. The LAD methods have been noticed to be attractive for real-life implementations, for example, in spectrum sensing in cognitive radios, because of their blindness, simplicity - their computational complexity is only $N \log _{2} N$ - and great performance [14]. However, most of the papers have concentrated on simulations in additive white Gaussian noise (AWGN) channels, so there is lack of results in realistic channels.

Heretofore, multipath situations have been considered only in [15]. Therein, snapshots were presented in the situations when there were one or two multipath components with fixed phase shifts and no Doppler. However, it is commonly known that real-life channels have much more multipath components with random phase shifts, fading and possible Doppler [16], which makes the situation very challenging. In this paper, we are using Elektrobit (EB) Propsim F8 radio channel emulator [17] for realistic channel modelling. ETSI Broadband Radio Access Network (BRAN)/ Wireless Local Area Network (WLAN) channel models B and C are used, so there are 17 multipath components with random phase shifts, Doppler effect and fading [18]. In addition, long measurements are performed to get statistical information. The detection performance of the FCME algorithm and the LAD methods in realistic multipath channels is of interest. Detection losses caused by model $\mathrm{B}$ and $\mathrm{C}$ channels as well as false alarm probabilities were studied. The probability of detection means the probability of a signal sample being classified as a signal sample, whereas the probability of false alarm means the probability of a noise-only sample being falsely classified as a signal sample. Although WLAN signal at $2.43 \mathrm{GHz}$ was used, the results are generalizable to other signals and frequency areas, because the FCME algorithm and the LAD methods are robust to the studied signal type and frequency area [14] 
According to the authors best knowledge, this is the first time when statistical information about the performance of the FCME, LAD ACC and 2-D LAD ACC methods under realistic channel conditions is presented.

\section{THE LAD METHODS}

The LAD methods are iterative outlier (=signal) detection methods that use two thresholds to find signals. Usually, the methods are used in the frequency domain. The thresholds are calculated using the FCME algorithm [19].

The iterative FCME algorithm is computationally simple but effective forward-type method that calculates the detection threshold based on the noise properties. The FCME algorithm operates as follows:

Step 1: Calculate the used threshold parameter

$$
T_{C M E}=-\ln \left(P_{F A, D E S}\right),
$$

where $P_{F A, D E S}$ is the desired clean sample rejection rate, i.e., the required false alarm rate [14]. For example, if the desired clean sample rejection rate is $1 \%\left(=10^{-2}\right), T_{C M E}=4.6052$. Step 2: Rearrange squared samples $\left|x_{i}\right|^{2}$ in an ascending order according to the sample energy and select $m=10 \%$ smallest samples to form the initial set $Q$.

Step 3: Calculate threshold

$$
T_{h}=T_{C M E} * \bar{Q},
$$

where - denotes the sample mean.

Step 4: Add samples $x^{2}<T_{h}$ to the set $Q$.

Repeat steps 3 and 4 until there are no new samples below the threshold. As a result, samples above the threshold are assumed to be signal samples and samples below the threshold are assumed to be noise samples.

The equation (1) for the threshold parameter $T_{C M E}$ is valid when the noise is at least approximately Gaussian, so the variable $\left|x_{i}\right|^{2}$ follows a chi-squared distribution with two degrees of freedom, i.e., magnitude-squared samples (energy) is considered. However, it is possible to define the equation to other distributions [14].

The LAD method [20] uses two FCME-thresholds called the upper and lower thresholds. Usually, the thresholds are calculated running the FCME algorithm twice with two different threshold parameters. The thresholds can also be set manually, but the drawback is that then the false alarm rate is not controlled if the noise level is not known. However, it depends on the situation if it is required or not. Here, we calculated the upper and lower thresholds as in [21]. The FCME algorithm was performed only once using one threshold parameter $T_{C M E}=4.6052$ [19]. After that, we used the mean $\bar{Q}$ from the obtained threshold (2) and added $6 \mathrm{~dB}$ to get the lower threshold and $10 \mathrm{~dB}$ to get the upper and FCME thresholds. These values approximately correspond $T_{C M E}=4$ and 10 , respectively. In this way, the thresholds are not too sensitive to the changes in the noise. This approach is similar to the normalized threshold (NT) threshold setting approach proposed in [22]. The LAD method groups the adjacent samples above the lower threshold and makes a test:

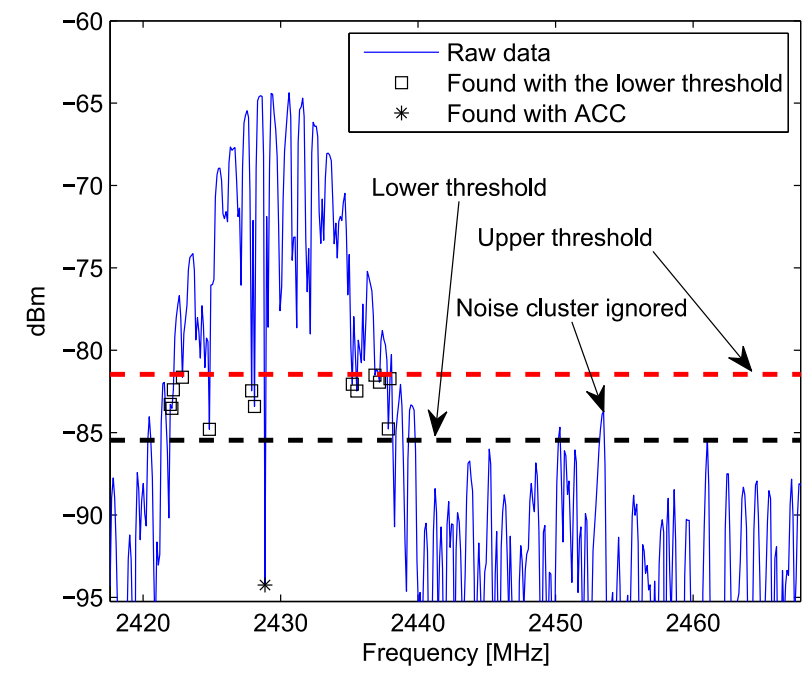

Fig. 1. The LAD ACC method, 802.11b signal with $11 \mathrm{Mbit} / \mathrm{sec}$ data rate, total packet size 1508 bytes, AWGN channel, channel emulator output level $-70 \mathrm{dBm}$.

if the largest element of the group is also above the upper threshold, the group corresponds a signal. Otherwise, the group is from the noise. The main point is to avoid falsely separated and falsely detected signals.

The LAD ACC method [22] adds an extra test after the LAD processing. That is, if two signal groups are close to each other, i.e., there are only $n$ noise samples between the signal groups, the signal groups are merged together to form one signal group. Here, $n=8$. This reduces the frequency fluctuation. The LAD ACC method is illustrated in Fig. 1.

The 2-D LAD method [23] corresponds binary detection in radar systems [24]. That is, $r$ consecutive time instants ('sweeps') are considered. The 2-D LAD method combines $r$ consecutive LAD (or LAD ACC) results in the time domain. One frequency domain sample is considered in time. If a frequency domain sample belongs to the signal at least $p$ times out of $r$ time instants, the frequency domain sample is decided to belong to the signal. This reduces the time fluctuation.

Blind signal detection is a demanding task. Even though the average signal spectrum has a clear shape, the shape of instantaneous spectrum fluctuates. The difficulty is emphasized when the signal power is weak, and when there are multipath components that cause constructive/destructive phenomenon yielding peaks and holes (notches) in the spectra. This problem is illustrated in Figs. 2 and 3. The whole $22 \mathrm{MHz}$ bandwidth includes also the sidelobes. Detection of the main lobe is much easier case because the sidelobes do not need to be detected. In addition, the narrower the signal the better the LAD methods operate [14]. Here, it is defined that the main lobe has $10 \mathrm{MHz}$ bandwidth. These two cases are illustrated in Fig. 3.

\section{Measurement Setup}

In the measurements we used Agilent E4438C ESG vector signal generator (Fig. 4) and Agilent Signal Studio Software 


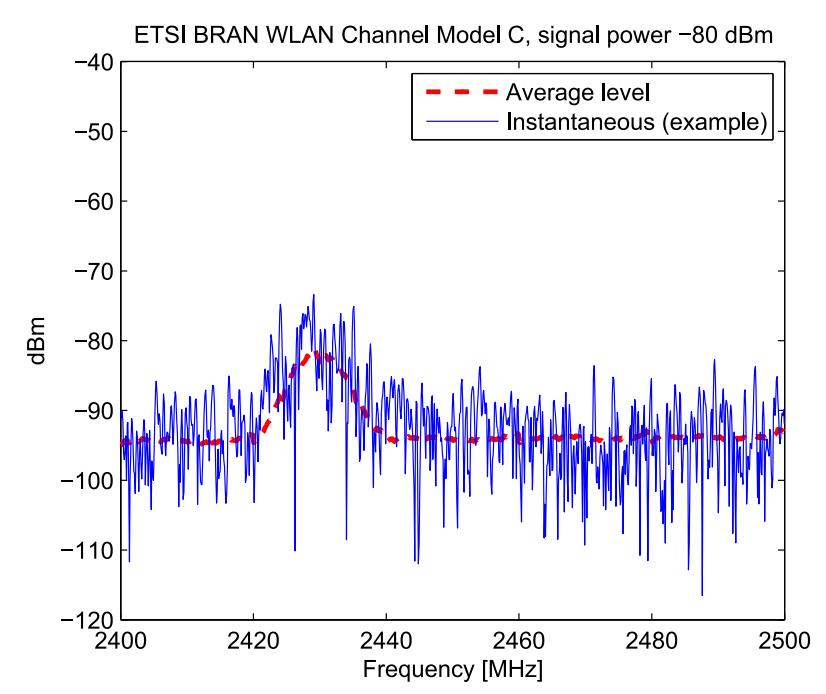

Fig. 2. WLAN signal at $2.43 \mathrm{GHz}$ and noise. Average and instantaneous levels. Signal power is $-80 \mathrm{dBm}$.

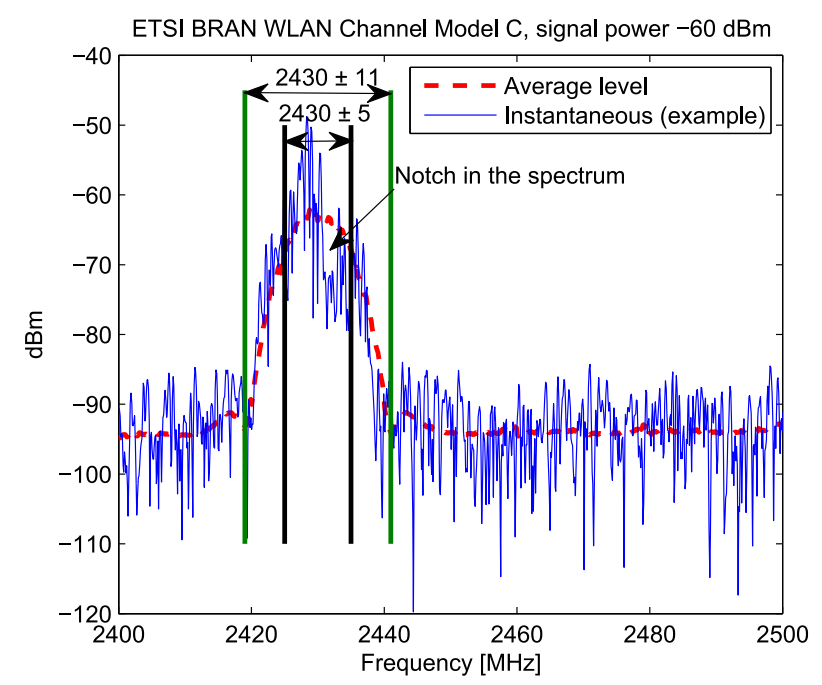

Fig. 3. WLAN signal at $2.43 \mathrm{GHz}$ and noise. Average and instantaneous levels. Signal power is $-60 \mathrm{dBm}$. The whole bandwidth is $22 \mathrm{MHz}$ and the width of the main lobe is defined to be $10 \mathrm{MHz}$.

to produce WLAN signal at $2.43 \mathrm{GHz}$ with idle time zero, i.e, we used continuous signal. The WLAN signal power was, on average, $-60,-70$ or $-80 \mathrm{dBm}$. EB Propsim F8 radio channel emulator (Fig. 5) was used for channel modelling [17]. The measurement instrument was Agilent N6841A RF Sensor with Creowave ISM band filter and Mini-Circuits ZRL-3500 LNA. The studied bandwidth was $100 \mathrm{MHz}(2.4-2.5 \mathrm{GHz})$. WLAN channel models B and C [18] were used. Model B is for a typical large open space and office environments with NLOS conditions and $100 \mathrm{~ns}$ rms delays spread (Fig. 6). Model C is for a large open space in indoor and outdoor with NLOS conditions and $150 \mathrm{~ns}$ rms delay spread (Fig. 7). In models $\mathrm{B}$ and $\mathrm{C}$, there were Doppler corresponding $5 \mathrm{~km} /$ hour device speed. Classical Rayleigh fading model was used [25]. AWGN channel was used as a point of comparison. In the 2-D LAD

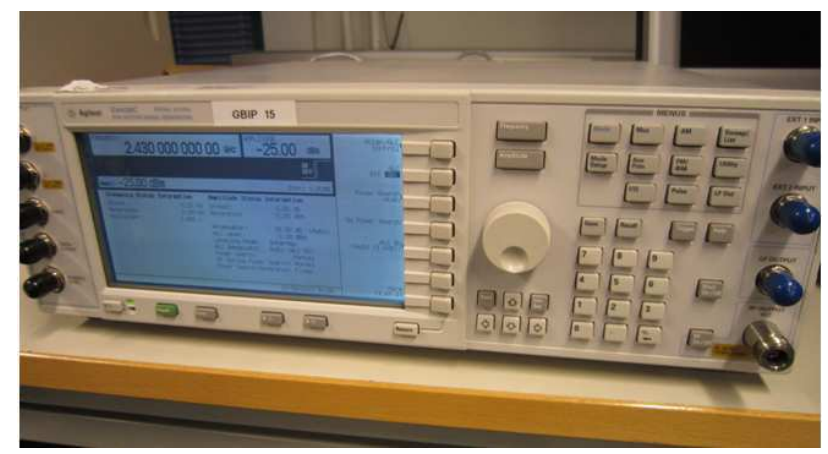

Fig. 4. Agilent E4438C ESG vector signal generator.

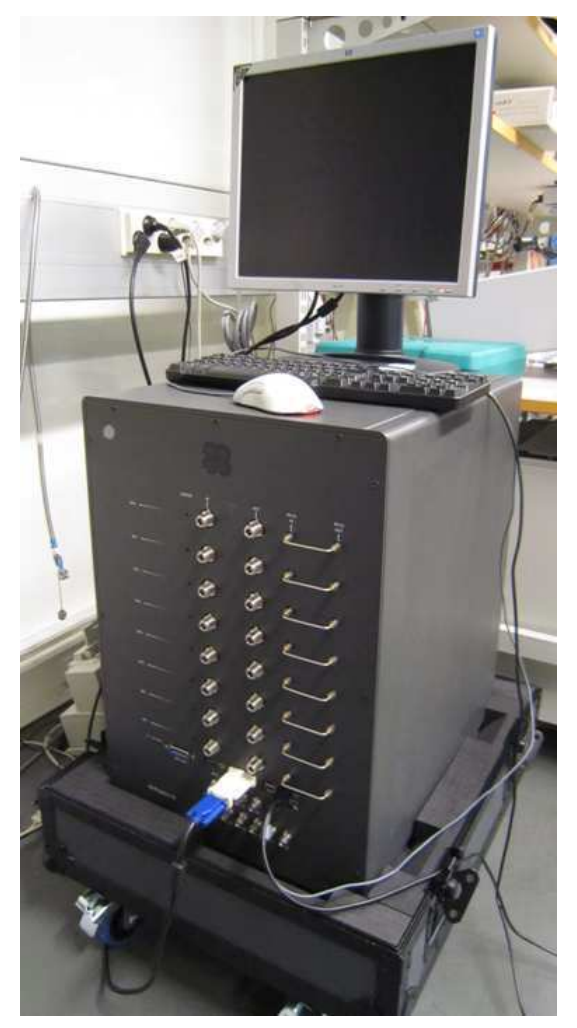

Fig. 5. EB Propsim F8 radio channel emulator.

ACC method, three different parameter combinations were used: $p=1$ and $r=5, p=1$ and $r=10, p=2$ and $r=5$. The length of each measurement was about 10 minutes. There were 100 sweeps per second and 916 frequency bins per every sweep, and the impulse response was continuously changing about 50 times per second. It was defined that the transmission time, or signal duration (='height') and frequencies (='width') of the transmitted signal constitutes the signal area. Thus, the signal is detected perfectly (detection performance 100\%) if all the time-frequency elements inside the signal area are classified as signal elements. Two different situations are considered: the whole $22 \mathrm{MHz}$ bandwidth $(22 \%$ of the studied band) or only the main lobe with $10 \mathrm{MHz}$ bandwidth (10\% of the studied band) is detected. 


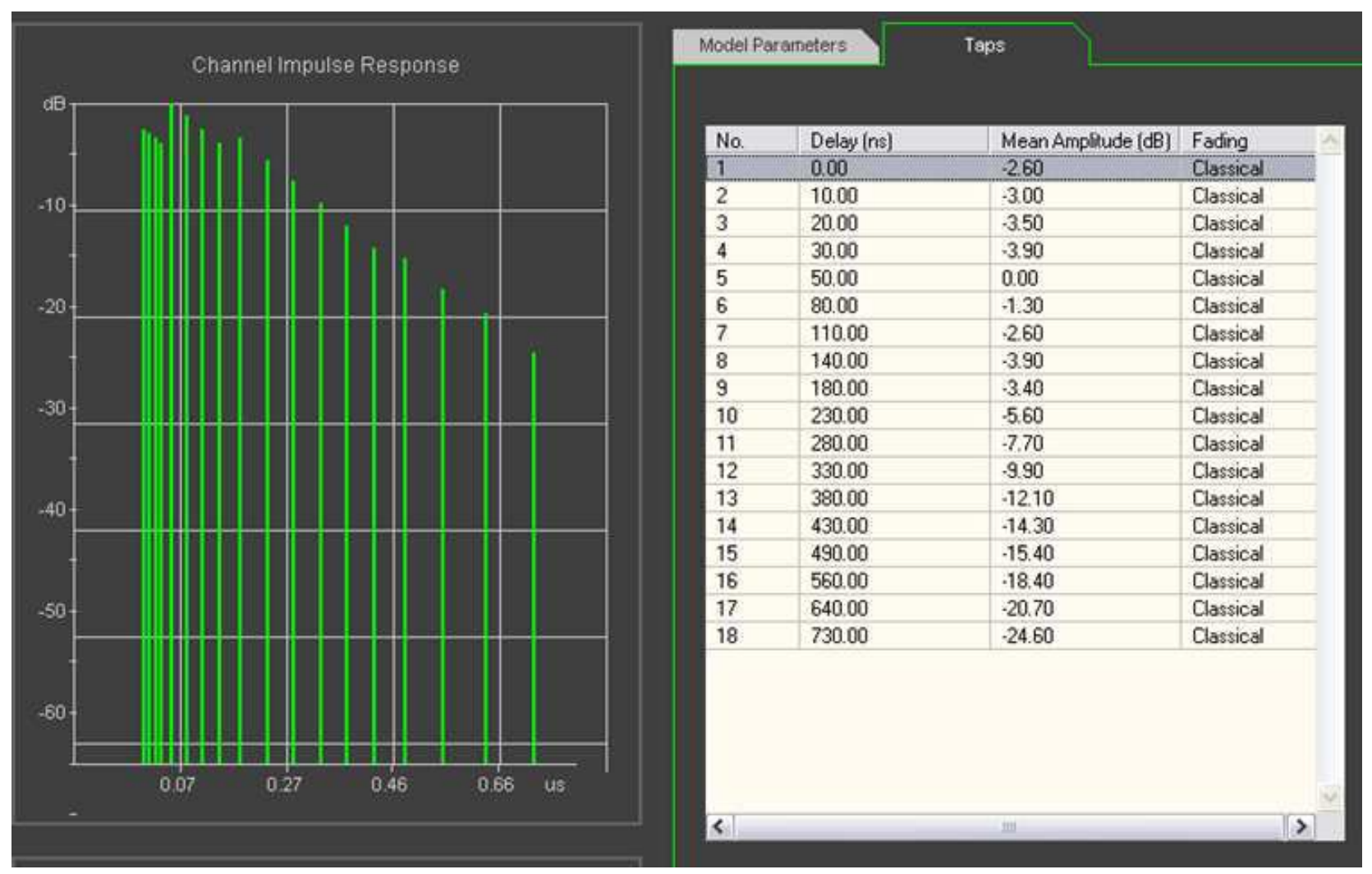

Fig. 6. ETSI BRAN WLAN channel model B. Impulse response, delays and mean amplitudes.

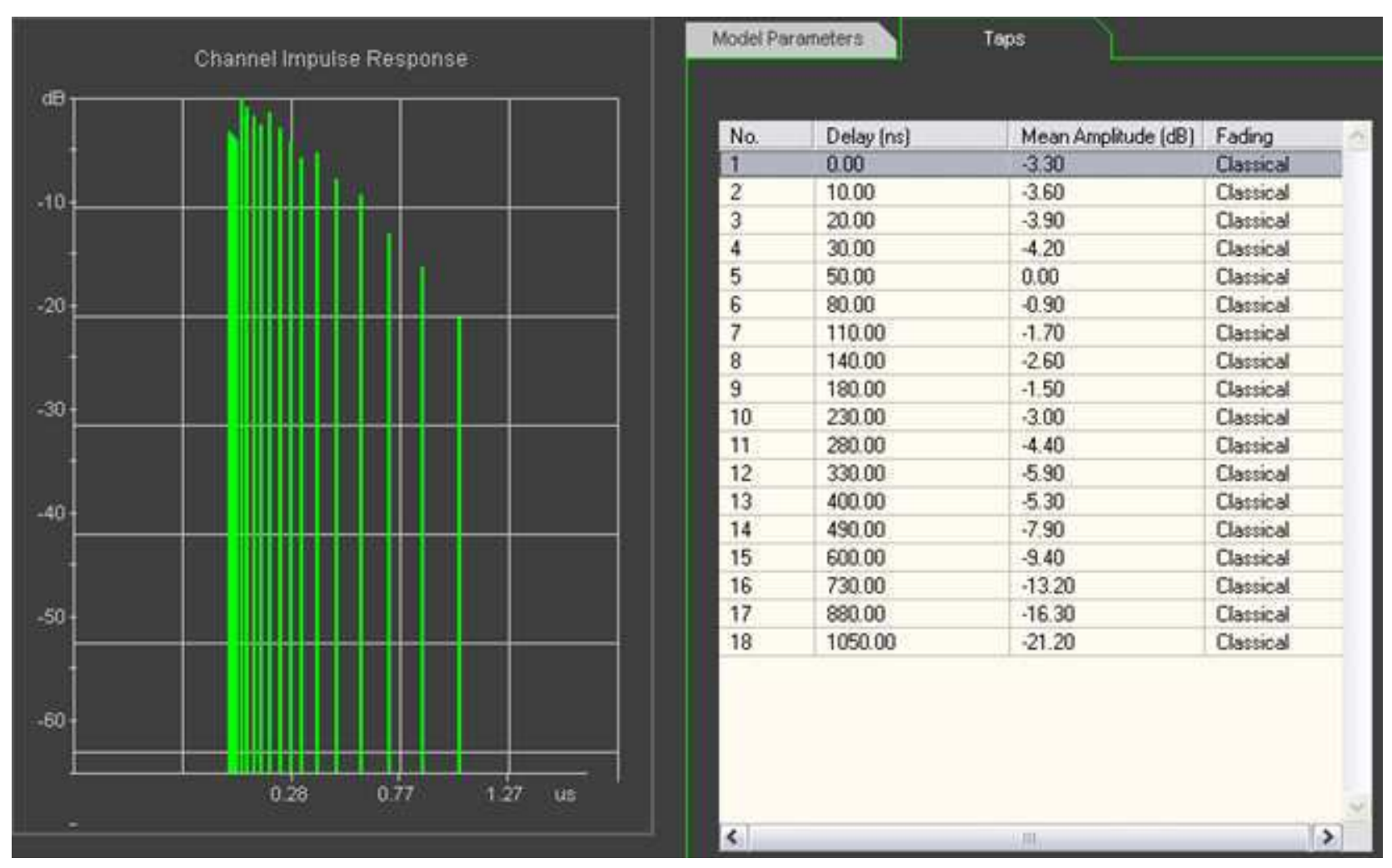

Fig. 7. ETSI BRAN WLAN channel model C. Impulse response, delays and mean amplitudes. 
TABLE I

Measurement results. Detection performance [\%] of the whole Wlan channel (BW 22 MHz) in AWGN, Model B AND Model C CHANNELS. SigNAL POWER IS $-60,-70$ AND -80 DBM.

\begin{tabular}{|c|c|c|c|c|c|c|c|c|c|}
\hline Method & $-60 \mathrm{dBm}$ & $\begin{array}{l}\text { AWGN } \\
-70 \mathrm{dBm}\end{array}$ & $-80 \mathrm{dBm}$ & $-60 \mathrm{dBm}$ & $\begin{array}{l}\text { Model B } \\
-70 \mathrm{dBm}\end{array}$ & $-80 \mathrm{dBm}$ & $-60 \mathrm{dBm}$ & $\begin{array}{l}\text { Model C } \\
-70 \mathrm{dBm}\end{array}$ & $-80 \mathrm{dBm}$ \\
\hline FCME & $79.9 \%$ & $60.9 \%$ & $27.1 \%$ & $74.7 \%$ & $52.4 \%$ & $21.3 \%$ & $74.9 \%$ & $52.6 \%$ & $21.0 \%$ \\
\hline LAD ACC & $87.0 \%$ & $74.6 \%$ & $48.7 \%$ & $83.9 \%$ & $68.0 \%$ & $37.2 \%$ & $84.2 \%$ & $68.7 \%$ & $37.3 \%$ \\
\hline 2-D LAD ACC $p=1, r=5$ & $90.6 \%$ & $80.6 \%$ & $59.8 \%$ & $89.9 \%$ & $79.1 \%$ & $57.1 \%$ & $90.1 \%$ & $79.3 \%$ & $57.1 \%$ \\
\hline 2-D LAD ACC $p=1, r=10$ & $91.7 \%$ & $82.6 \%$ & $63.3 \%$ & $91.8 \%$ & $82.4 \%$ & $63.5 \%$ & $91.9 \%$ & $82.5 \%$ & $63.2 \%$ \\
\hline 2-D LAD ACC $p=2, r=5$ & $88.7 \%$ & $77.2 \%$ & $53.6 \%$ & $87.3 \%$ & $74.5 \%$ & $48.0 \%$ & $87.4 \%$ & $74.7 \%$ & $48.1 \%$ \\
\hline
\end{tabular}

TABLE II

Measurement Results. Detection Performance [\%] of THE MAin LOBE of The WLAN CHANNEL (BW 10 MHz) In AWGN, Model B AND Model C CHANNELS. Signal POWER IS $-60,-70$ AND -80 DBM.

\begin{tabular}{|c|c|c|c|c|c|c|c|c|c|}
\hline \multirow[b]{2}{*}{ Method } & \multicolumn{3}{|c|}{ AWGN } & \multicolumn{3}{|c|}{ Model B } & \multicolumn{3}{|c|}{ Model C } \\
\hline & $-60 \mathrm{dBm}$ & $-70 \mathrm{dBm}$ & $-80 \mathrm{dBm}$ & $-60 \mathrm{dBm}$ & $-70 \mathrm{dBm}$ & $-80 \mathrm{dBm}$ & $-60 \mathrm{dBm}$ & $-70 \mathrm{dBm}$ & $-80 \mathrm{dBm}$ \\
\hline FCME & $98.5 \%$ & $92.7 \%$ & $54.8 \%$ & $96.2 \%$ & $82.5 \%$ & $41.7 \%$ & $96.5 \%$ & $83.0 \%$ & $41.2 \%$ \\
\hline LAD ACC & $100 \%$ & $99.9 \%$ & $94.4 \%$ & $99.9 \%$ & $97.6 \%$ & $70.2 \%$ & $99.9 \%$ & $98.4 \%$ & $70.7 \%$ \\
\hline 2-D LAD ACC $p=1, r=5$ & $100 \%$ & $100 \%$ & $99.9 \%$ & $100 \%$ & $99.9 \%$ & $96.2 \%$ & $100 \%$ & $99.9 \%$ & $96.6 \%$ \\
\hline 2-D LAD ACC $p=1, r=10$ & $100 \%$ & $100 \%$ & $99.9 \%$ & $100 \%$ & $99.9 \%$ & $99.5 \%$ & $100 \%$ & $99.9 \%$ & $99.6 \%$ \\
\hline 2-D LAD ACC $p=2, r=5$ & $100 \%$ & $100 \%$ & $99.8 \%$ & $100 \%$ & $99.9 \%$ & $89.4 \%$ & $100 \%$ & $99.9 \%$ & $90.0 \%$ \\
\hline
\end{tabular}

\section{Measurement Results}

The detection performance of the FCME algorithm and the LAD ACC and the 2-D LAD ACC methods are presented in Tables I-IV. We are interested to know how the challenging, realistic channels with multipath, fading and Doppler affect to the performance of those method. The detection loss under the studied channel model is given with respect to AWGN channel. As already known, the 2-D LAD ACC method outperforms the LAD ACC method which outperforms the LAD method which outperforms the FCME algorithm [14]. The LAD ACC method is a direct extension of the original LAD method. Thus, the performance of the original LAD method is not studied here.

Based on the measurement results we can make some general remarks. It can be said that the stronger the signal is, the better the detection capability is, as expected. There are only minor differences in the detection results between channel models $\mathrm{B}$ and $\mathrm{C}$. The third remark is that the 2-D LAD ACC method with parameters $p=2, r=5$ had the worst detection performance and there was no major difference between the results we got using parameters $p=1, r=5$ and $p=1, r=10$. Thus, $p=1, r=5$ and $p=1, r=10$ can be said to be proper choices. With parameter $r=5$ the method adapts more rapidly to the changes in the environment (signal present/not) [23].

Detection performance results when detecting the whole $22 \mathrm{MHz}$ bandwidth ( $22 \%$ of the studied band) are presented in Table I and detection performance results when detecting only the main lobe with $10 \mathrm{MHz}$ bandwidth (10\% of the studied band) are presented in Table II. For example, we can see from Table I that when the signal power is $-60 \mathrm{dBm}$, the detection loss caused by the realistic model $\mathrm{B}$ and $\mathrm{C}$ channels for the FCME algorithm is 5.2 (model B channel) and 5 (model $\mathrm{C}$ channel) in percentage points. It can be seen that the LAD methods perform very well. When the signal power is relatively high $(-60 \mathrm{dBm})$, the difference between
TABLE III

Measurement Results. Detection loss [\%] In Model B And MODEL C CHANNELS ON AVERAGE WHEN COMPARED TO THE DETECTION RESULTS IN AWGN CHANNELS. THE WHOLE WLAN CHANNEL (BW 22 MHZ) IS DETECTED.

\begin{tabular}{l||l|l|l} 
Method & $-60 \mathrm{dBm}$ & $-70 \mathrm{dBm}$ & $-80 \mathrm{dBm}$ \\
\hline FCME & $6 \%$ & $14 \%$ & $22 \%$ \\
LAD ACC & $3 \%$ & $8 \%$ & $24 \%$ \\
2-D LAD ACC, $p=1, r=5$ & $<1 \%$ & $2 \%$ & $5 \%$ \\
2-D LAD ACC, $p=1, r=10$ & - & $<1 \%$ & - \\
2-D LAD ACC, $p=2, r=5$ & $2 \%$ & $13 \%$ & $11 \%$ \\
\hline - no effect & \multicolumn{2}{l}{}
\end{tabular}

TABLE IV

Measurement Results. Detection loss [\%] In Model B And MOdEL C CHANNELS ON AVERAGE WHEN COMPARED TO THE DETECTION RESULTS IN AWGN CHANNELS. THE MAIN LOBE OF THE WLAN CHANNEL (BW $10 \mathrm{MHZ)} \mathrm{IS} \mathrm{DETECTED.}$

\begin{tabular}{l||l|l|l} 
Method & $-60 \mathrm{dBm}$ & $-70 \mathrm{dBm}$ & $-80 \mathrm{dBm}$ \\
\hline FCME & $2 \%$ & $10 \%$ & $25 \%$ \\
LAD ACC & $<1 \%$ & $2 \%$ & $25 \%$ \\
2-D LAD ACC, $p=1, r=5$ & - & $<1 \%$ & $4 \%$ \\
2-D LAD ACC, $p=1, r=10$ & - & $<1 \%$ & $4 \%$ \\
2-D LAD ACC, $p=2, r=5$ & - & $<1 \%$ & $10 \%$ \\
\hline - no effect &
\end{tabular}

the LAD ACC and 2-D LAD ACC methods is only about $2-8$ percentage points. When the signal power is weak $(-80$ $\mathrm{dBm})$, the difference is about $5-26$ percentage points. When detecting the main lobe of the WLAN channel, the detected bandwidth is $10 \mathrm{MHz}$, so it is about half of the channel. It can be noticed from Table II that the 2-D LAD ACC has excellent performance with almost perfect detection regardless of the signal power. This is due to the facts that the sidelobes that have lower signal power level are not detected, and the detected signal is narrower than in the precious case with respect to the studied bandwidth [12]. 

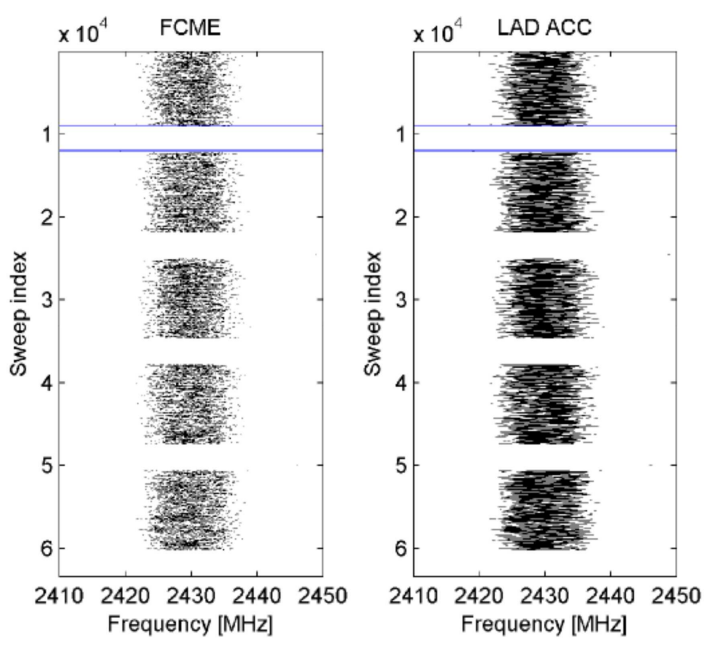

Fig. 8. WLAN signal at $2.43 \mathrm{GHz}$ in AWGN channel. Signal power is -80 $\mathrm{dBm}$. One cycle duration was 2 minutes. Detection using the FCME and the LAD ACC methods.

In Tables III and IV, detection loss results are presented. It can be noted that with proper 2-D LAD ACC parameters, detection losses [\%] are, an average, on the same level when detecting the whole WLAN channel or only the main lobe. For example, when the LAD ACC method is used, the detection loss caused by channel models $\mathrm{B}$ and $\mathrm{C}$ is 3 or $<1 \%$ when the WLAN signal power is $-60 \mathrm{dBm}$. It can be noticed that the stronger the signal is the less there is detection loss. With weaker signal, the detection losses are higher. When the 2-D LAD ACC method with proper parameters is used, model B and $\mathrm{C}$ channels weaken the detection performance only $0-5 \%$.

Good detection performance is not enough. To ensure good total performance, it is required that the number of falsely detected signals is low. Here, pulsed WLAN signal was used to define probabilities of false alarms $\left(P_{f a}\right)$. In Fig. 8, the received spectrogram of one snapshot is presented. There were 60000 sweeps. One cycle duration was 2 minutes $(90 \mathrm{sec}$. transmission and $30 \mathrm{sec}$. silence) and there were five repetitions. Based on the silent periods, we got $P_{f a}=1.3 \cdot 10^{-4}$ for the FCME algorithm and $P_{f a}=3.6 \cdot 10^{-4}$ for the LAD ACC method. These values correspond $T_{C M E}=9$ and 8 (1). These probabilities are valid also in model $\mathrm{B}$ and $\mathrm{C}$ channels. It can be concluded that the false alarm probabilities are low to both the FCME algorithm and the LAD methods.

\section{CONCLUSIONS}

The performance of the LAD methods in realistic ETSI BRAN/WLAN channel models B and C was studied. It was noticed that when compared to the results in AWGN channel, the LAD ACC method has $1-3 \%$ loss in the detection performance when the signal power is high, and the 2-D LAD ACC method has at most $5 \%$ detection loss regardless of the signal power. In addition, false alarm probabilities are low. Thus, it can be said that the LAD methods can be used in real-life applications.

\section{REFERENCES}

[1] S. Haykin, "Cognitive radio: Brain-empowered wireless communications," IEEE Journal in Selected Areas in Comm., vol. 23, no. 2, pp. 201-220, Feb. 2005.

[2] A. Goldsmith, S. A. Jafar, I. Maric, and S. Srinivasa, "Breaking spectrum gridlock with cognitive radios: An information theoretic perspective," IEEE Proceedings, vol. 97, no. 5, pp. 894-914, 2009.

[3] FCC, "FCC frees up vacant TV airwaves for super Wi-Fi technologies," Sept. 2010, http://www.fcc.gov.

[4] J. Mitola III and G. Q. Maguire Jr., "Cognitive radio: making software radios more personal,” IEEE Pers. Commun., vol. 6, no. 4, pp. 13-18, 1999.

[5] J. Mitola III, "Cognitive radio architecture evolution," IEEE Proceedings, vol. 97, no. 4, pp. 626-641, 2009.

[6] C. Cordeiro, K. Challapali, D. Birru, and S. N. Shankar, "Spectrum agile radios: Utilization and sensing architectures," in DYSPAN, Baltimore, USA, Nov. 2005, vol. 1, pp. 328-337.

[7] A. Sahai, D. Cabric, N. Hoven, R. Tandra, S.M. Mishra, and R. Brodersen, "Spectrum sensing: fundamental limits and practical challenges (tutorial)," in DYSPAN, Baltimore, Maryland, Nov. 2005.

[8] R. Fan and H. Jiang, "Optimal multi-channel cooperative sensing in cognitive radio networks," IEEE Trans. on Wireless Commun., vol. 9, no. 3, pp. 1128-1138, March 2010.

[9] S. M. Kay, Fundamentals of statistical signal processing: Detection theory, Prentice Hall, Upple Saddle River, NJ, USA, 1998.

[10] H. L. Van Trees, Detection, Estimation, and Modulation Theory, Part I, John Wiley, New York, USA, 1968.

[11] H. V. Poor, An introduction to signal detection and estimation, SpringerVerlag, Berlin, Germany, 2nd edition, 1998.

[12] J. Vartiainen, J. J. Lehtomäki, H. Saarnisaari, and M. Juntti, “Analysis of the consecutive mean excision algorithms," J. Elect. Comp. Eng., 2011.

[13] T. Hänninen, J. Vartiainen, M. Juntti, and M. Raustia, "Implementation of spectrum sensing on wireless open-access research platform," in CogART 2010, Rome, Italy, Nov. 2010.

[14] J. Vartiainen, Concentrated Signal Extraction Using Consecutive Mean Excision Algorithms, Ph.D. thesis, Acta Univ Oul Technica C 368. Faculty of Technology, University of Oulu, Finland, Nov. 2010, http: //jultika.oulu.fi/Record/isbn978-951-42-6349-1.

[15] J. Vartiainen, J. Lehtomäki, and R. Vuohtoniemi, "Performance of the LAD methods under channel impairments," in CogArt 2011, Barcelona, Spain, Oct. 2011.

[16] Cisco Systems, "Multipath and diversity," in Document ID: 27147, 2008, http://www.cisco.com/application/pdf/paws/27147/multipath.pdf.

[17] EB Propsim F8, ," http://www.elektrobit.com/what_we_deliver/wireless/ offering/propsim_f8.

[18] J. Medbo and P. Schramm, "Channel models for HIPERLAN/2," ETSI/BRAN document no. 3ERI085B.

[19] H. Saarnisaari, P. Henttu, and M. Juntti, "Iterative multidimensional impulse detectors for communications based on the classical diagnostic methods," IEEE Trans. Commun., vol. 53, no. 3, pp. 395-398, March 2005.

[20] J. Vartiainen, J. J. Lehtomäki, and H. Saarnisaari, "Double-threshold based narrowband signal extraction," in VTC 2005, Stockholm, Sweden, May/June 2005

[21] J. J. Lehtomäki, R. Vuohtoniemi, K. Umebayashi, and J.-P. Mäkelä, "Energy detection based estimation of channel occupancy rate with adaptive noise estimation," IEICE Trans. Commun., vol. E95-B, no. 04, Apr. 2012.

[22] J. Vartiainen, H. Sarvanko, J. Lehtomäki, M. Juntti, and M. Latva-aho, "Spectrum sensing with LAD based methods," in PIMRC 2007, Athens, Greece, Aug. 2007.

[23] J. Vartiainen, J. J. Lehtomäki, H. Saarnisaari, and M. Juntti, "Twodimensional signal localization algorithm for spectrum sensing," IEICE Trans. Commun., vol. E93-B, no. 11, pp. 3129-3136, Nov. 2010.

[24] M. I. Skolnik, Introduction to Radar Systems, McGraw-Hill, NY, USA, 2001.

[25] W. C. Jakes, Ed., Microwave mobile communications, Wiley, New York, USA, 1974. 